\title{
Aspectos epidemiológicos do vírus da dengue em mulheres grávidas no Estado do Pará,
}

\section{no período de 2015 a 2019}

Epidemiological aspects of the dengue virus in pregnant women in the State of Pará, from 2015 to 2019

Aspectos epidemiológicos del virus del dengue en gestantes del Estado de Pará, de 2015 a 2019

Marcela Raíssa Asevedo Dergan ORCID: https://orcid.org/0000-0003-1457-0242

Escola Superior da Amazônia, Brasil

E-mail: derganm20@gmail.com

Lilya Helena Casanova Pereira Melo ORCID: https://orcid.org/0000-0002-7429-0253

Escola Superior da Amazônia, Brasil E-mail: lilyacasanova01@gmail.com

Tawana Nogueira Chagas

ORCID: https://orcid.org/0000-0003-1023-9907

Escola Superior da Amazônia, Brasil

E-mail: tawchagas@gmail.com

Dayara de Nazaré Rosa de Carvalho

ORCID: https://orcid.org/0000-0001-8569-3392

Universidade do Estado do Pará, Brasil

E-mail: dayara_twain@hotmail.com

Analdo Costa Alfaia Júnio

ORCID: https://orcid.org/0000-0002-9057-730X

Faculdade Finama, Brasil

E-mail: jr_supersom@hotmail.com

Thales Silva

ORCID: https://orcid.org/0000-0002-7198-070X

Faculdade Faci, Brasil

E-mail: thalesilva999@yahoo.com

Ellen Rejane Lima da Conceição Rodrigues

ORCID: https://orcid.org/0000-0002-2457-4100

Faculdade Finama, Brasil

E-mail: ellenlima52@hotmail.con

Gabriela Nunes Pinheiro

ORCID: https://orcid.org/0000-0002-0126-3510

Universidade da Amazônia, Brasil

E-mail: james.silva008@gmail.com

Eliana Caldas Barbosa

ORCID: https://orcid.org/0000-0002-9530-7464

Faculdade Finama, Brasil

E-mail:ana.acndp@hotmail.com

Paula Sousa da Silva Rocha

ORCID: https://orcid.org/0000-0002-0453-1314

Universidade Federal do Pará, Brasil

E-mail: paulatuc@msn.com

Vera Regina da Cunha Menezes Palácios

ORCID: https://orcid.org/0000-0003-0300-7972

Universidade do Estado do Pará, Brasil

E-mail: verareginapalacios@gmail.com

Fabíolla da Silva dos Santos

ORCID: https://orcid.org/0000-0001-6317-0988

Escola Superior da Amazônia, Brasil

E-mail: fabisavir@gmail.com

\section{Resumo}

O presente estudo teve como objetivo avaliar os aspectos epidemiológicos da infecção pelo vírus da Dengue em mulheres grávidas no Estado do Pará, identificando os principais fatores que acarretam na incidência da doença em gestantes. Metodologia: Trata-se de um estudo quantitativo, retrospectivo, descritivo-exploratório com base nos dados 
epidemiológicos fornecidos pelo Departamento de Informática do Sistema de Saúde Unificado (DATASUS) no período de 2015 a 2019, nas regiões de integração do Estado do Pará. Resultados e discussão: Foram identificados 2.397 casos de dengue em mulheres grávidas durante o período descrito com um aumento de casos no período de 2015 a 2016 , reduções entre 2016 a 2018 e uma nova elevação no ciclo 2018 a 2019. De acordo com a pesquisa, em relação ao perfil sócio-demográfico, a doença recebeu mais notificações em grávidas que estavam no segundo trimestre gestacional, na faixa etária de 20 a 39 anos e com respectivos casos em autóctones do município de residência. Percebe-se um maior número de casos da doença nas Regiões de Saúde do Carajás, Metropolitana I, Xingu, Araguaia e Rio Caetés. Considerações finais: A dengue em gestantes no Pará prossegue existindo como uma doença negligenciada a qual necessita de ações que visem preveni-la entre esse público, e que incentivem uma maior compreensão da doença entre os profissionais de saúde envolvidos. Mais estudos são necessários para a atualização dos dados clínico-epidemiológicos acerca da dengue e seus agravos.

Palavras-chave: Dengue; Gravidez; Epidemiologia; Saúde pública.

\begin{abstract}
This study aimed to evaluate the epidemiological aspects of dengue virus infection in pregnant women in the state of Pará, identifying the main factors that lead to the incidence of the disease in pregnant women. Methodology: This is a quantitative, retrospective, descriptive-exploratory study based on epidemiological data provided by the Unified Health System Informatics Department (DATASUS) from 2015 to 2019, in the integration regions of the State of Pará. 2,397 cases of dengue were identified in pregnant women during the period described with an increase in cases from 2015 to 2016, reductions between 2016 and 2018 and a new increase in the 2018 to 2019 cycle. Results and discussion: According to this research, in relation to the socio-demographic profile, the disease received more notifications in pregnant women who were in the second trimester of pregnancy, in the age group of 20 to 39 years and with respective cases in natives of the municipality of residence. There is a greater number of cases of the disease in the Health Regions of Carajás, Metropolitana I, Xingu, Araguaia and Rio Caetés. Final considerations: Dengue in pregnant women in Pará continues to exist as a neglected disease. For this reason, it demands the existence of actions aimed at preventing it among this public and at encouraging a greater understanding of the disease among the health professionals involved. More studies are needed to update the clinical-epidemiological data on dengue and their disorders.
\end{abstract}

Keywords: Dengue; Pregnancy; Epidemiology; Public health.

\title{
Resumen
}

El presente estudio tuvo como objetivo evaluar los aspectos epidemiológicos de la infección por el virus del dengue en mujeres embarazadas en el Estado de Pará, identificando los principales factores que implican en la incidencia de la enfermedad en gestantes. Metodología: Se trata de un estudio cuantitativo, retrospectivo, descriptivo-exploratorio basado en los datos epidemiológicos proporcionados por el Departamento de Informática del Sistema de Salud Unificado (DATASUS) en el período 2015 a 2019, en las regiones de integración del Estado de Pará. Resultados y discusíon: Se identificaron 2.397 casos de dengue en mujeres embarazadas durante el período descrito con un aumento de casos en el período de 2015 a 2016, reducciones entre 2016 y 2018 y una nueva elevación en el ciclo 2018 a 2019. En relación al perfil sociodemográfico, la enfermedad recibió más notificaciones en embarazadas que estaban en el segundo trimestre gestacional, en el grupo de edad de 20 a 39 años y con sus casos en autóctonas del municipio de residencia. Se detecta un mayor número de casos de la enfermedad en las Regiones de Salud del Carajás, Metropolitana I, Xingu, Araguaia y Río Caetés. Consideraciones finales: El dengue en gestantes en Pará continúa existiendo como una enfermedad olvidada que necesita acciones que busquen prevenirla entre ese público, y que incentiven una mayor comprensión de la enfermedad entre los profesionales de salud involucrados. Más estudios son necesarios para la actualización de los datos clínico-epidemiológicos sobre el dengue y sus agravios.

Palabras clave: Dengue; Embarazo; Epidemiología; Salud pública.

\section{Introdução}

A dengue é uma doença infecciosa que representa um grave problema de saúde pública no Brasil e no mundo, é transmitida pelo mosquito Aedes aegypti e, em menor grau, pelo Aedes albopictus, caracteriza-se por possuir quatro sorotipos (DEN-1, DEN-2, DEN-3, DEN-4), sendo o DEN-3 o mais virulento. A transmissão é associada principalmente com variações climáticas como o índice pluviométrico, temperatura, umidade relativa do ar, além de outros fatores como urbanização rápida e não planejada das cidades (Furtado et al.,2019). 
O Aedes aegypti, necessita de água parada para se proliferar e o período do ano de maior transmissibilidade são os meses de elevada pluviosidade de cada região, sendo fundamental manter as medidas de prevenção como higiene adequada e evitar acúmulo de água parada em locais propícios, pois os ovos do mosquito podem sobreviver por um ano até encontrar condições ideais para reprodução (Ministério da Saúde, 2019).

Nas últimas cinco décadas a incidência de dengue aumentou 30 vezes, e por volta de 2,5 bilhões de indivíduos estão em condições favoráveis ao adoecimento, principalmente em regiões tropicais e subtropicais, na década de 80 intensas epidemias já eram registradas em vários países, entre eles, o Brasil apresentava 14\% dos casos graves, mesmo percentual de todo o continente africano (Ministério da Saúde, 2015).

A doença pode afetar qualquer pessoa, porém as mulheres grávidas correm mais risco, uma vez que os sintomas clínicos comuns da doença como a febre, pode causar aborto ou parto prematuro. A trombocitopenia relacionada à dengue aumenta o risco de sangramento durante a gravidez ou no parto e, portanto, leva a uma maior taxa de mortalidade materna (Sondo et al.,2019).

No ciclo dos cuidados de saúde reprodutiva, os cuidados pré-natais (CPN) constituem uma plataforma para importantes funções dos cuidados de saúde, incluindo a promoção da saúde, o rastreio, o diagnóstico e a prevenção das doenças. Estas funções de comunicação e apoio aos CPN são essenciais, não só para salvar vidas, mas também para melhorar a vida, a utilização dos cuidados de saúde e a sua qualidade (Organização Mundial da Saúde, 2018).

Nesse contexto, realizar estudos sobre a dengue no ciclo gravídico é fundamental para atualização de casos e divulgar informações que venham a contribuir com as ações de vigilância epidemiológica no Estado do Pará. Diante disso, esta pesquisa tem como objetivo traçar o perfil clínico-epidemiológico da dengue em mulheres grávidas no Estado do Pará, no período de 2015 a 2019.

\section{Metodologia}

Trata-se de um estudo quantitativo, retrospectivo, descritivo-exploratório. Optou-se por uma abordagem quantitativa pela necessidade de realizar o levantamento de dados clínico epidemiológicos da dengue em pacientes grávidas no Estado do Pará.

Na pesquisa quantitativa utiliza-se a medição de grandezas que são obtidas por meio da metrologia, números com suas respectivas unidades, gerando conjuntos de dados que permitem ser analisados por meio de técnicas matemáticas (Pereira et al.,2018).

Foram utilizados os dados secundários registrados no Departamento de Informática do Sistema de Saúde Unificado (DATASUS), disponível no endereço eletrônico https://datasus.saude.gov.br/. Selecionaram-se os dados de casos diagnosticados de dengue em pacientes mulheres no ciclo gravídico, considerando-se a série histórica 2015-2019, nas Regiões de Integração do Estado do Pará.

Foram selecionadas as variáveis sócio-demográficas: ano, faixa etária, raça e município de residência, e as variáveis clínico-epidemiológicas: região de notificação (CIR) de residência, exame sorológico (IgM e ELISA), exame de isolamento viral, exame de histopatologia, sorotipo de dengue, classificação final e evolução do paciente.

A coleta de dados ocorreu no período de outubro e novembro de 2020, e em seguida houve o agrupamento dos dados selecionados no programa Microsoft Excel 2016 de forma a mantê-los organizados para o prosseguimento do estudo. Posteriormente, houve a análise descritiva por meio do software Biostat versão 5.3 onde se identificou os respectivos percentuais.

Por se tratar apenas de dados secundários e de domínio público, essa pesquisa não necessitou de envio ao Comitê de Ética em Pesquisa/ CEP-CONEP. 


\section{Resultados}

Identificou-se um total de 2.397 casos de dengue em mulheres grávidas no período de 2015 a 2019, nota-se um aumento no número de casos da doença no ano de 2015 a 2016, e respectivas reduções nos anos de 2016 a 2018, já no ciclo 2018 a 2019 observa-se uma nova elevação do número de casos notificados. Na Tabela 1 verifica-se a distribuição dos casos de dengue na gravidez por ano e mês de infecção.

Tabela 1. Distribuição dos casos notificados por ano e mês de infecção em mulheres grávidas infectadas pelo vírus dengue no Estado do Pará, no período de 2015 a 2019.

\begin{tabular}{lcc}
\hline \multicolumn{1}{c}{ Variáveis } & \multicolumn{2}{c}{ Total de casos= 2.397 } \\
\hline Ano & $\mathbf{N}^{\circ}$ & $\%$ \\
\hline 2015 & 42 & 1,75 \\
2016 & 292 & 12,1 \\
2017 & 149 & 6,2 \\
2018 & 79 & 3,3 \\
2019 & 90 & 3,75 \\
Ing/branco & 1.745 & 72,7 \\
Mês de Infecção & & \\
Janeiro & 69 & 2,8 \\
Fevereiro & 135 & 5,6 \\
Março & 105 & 4,3 \\
Abril & 76 & 3,1 \\
Maio & 60 & 2,5 \\
Junho & 31 & 1,3 \\
Julho & 41 & 1,7 \\
Agosto & 19 & 0,8 \\
Setembro & 39 & 1,6 \\
Outubro & 18 & 0,75 \\
Novembro & 28 & 1,1 \\
Dezembro & 31 & 1,2 \\
\hline
\end{tabular}

Fonte: Departamento de Informática do SUS/DATASUS.

Em relação ao perfil sócio-demográfico, nota-se que a doença recebeu mais notificações em grávidas que estavam no $2^{\circ}$ trimestre gestação, na faixa etária de 20 a 39 anos e com respectivos casos em autóctones do município de residência, enfatiza-se também as expressivas notificações ignoradas e em branco. Na Tabela 2 identifica-se o perfil sócio-demográfico de mulheres grávidas infectadas pelo vírus da dengue no Estado do Pará, no período de 2015 a 2019. 
Research, Society and Development, v. 10, n. 1, e20810111641, 2021

(CC BY 4.0) | ISSN 2525-3409 | DOI: http://dx.doi.org/10.33448/rsd-v10i1.11641

Tabela 2. Perfil sócio-demográfico de mulheres grávidas infectadas pelo vírus da dengue no Estado do Pará, no período de 2015 a 2019.

\begin{tabular}{lcc}
\hline \multicolumn{1}{c}{ Variáveis } & Total de casos $=\mathbf{2 . 3 9 7}$ \\
\hline Idade Gestacional & $\mathbf{N}^{\mathbf{0}}$ & $\mathbf{\%}$ \\
$1^{\mathbf{0}}$ trimestre & 172 & 7,1 \\
$2^{\mathbf{o}}$ trimestre & 249 & 10,3 \\
$3^{\circ}$ trimestre & 231 & 9,6 \\
Idade gestacional Ignorada & 72 & 3 \\
Faixa etária & & \\
$10-14$ & 15 & 0,6 \\
$15-19$ & 139 & 5,7 \\
$20-39$ & 485 & 20,2 \\
Não se aplica & 121 & 5 \\
Ign/Branco & 287 & 11,9 \\
Raça & & \\
Branca & 112 & 4,6 \\
Preta & 31 & 1,3 \\
Parda & 479 & 20 \\
Amarela & 02 & 0,08 \\
Indígena & 02 & 0,08 \\
Autóctone município de residência & & 0,4 \\
Sim & 277 & 15,1 \\
Não & 10 & 0,12 \\
Em branco & 362 & 1,5 \\
Indeterminado & 3 & 0,4 \\
\hline
\end{tabular}

Fonte: Departamento de Informática do SUS/DATASUS.

O perfil clínico-epidemiológico caracterizou-se pela recorrência de casos nas Regiões de Saúde dos Carajás, seguido das regiões Metropolitana I, Xingu, Araguaia e Rio Caetés. No que diz respeito à classificação final e a evolução do paciente, observou-se que grande parte dos casos é de dengue e dengue clássico, havendo poucos casos da doença com sinais de alarme e nenhum de febre hemorrágica decorrente da infecção pelo vírus.

Em relação ao diagnóstico, observou-se 83 casos positivos nos exames sorológicos de IgM e ELISA, porém há mais notificações que indicam a não realização das sorologias e do exame histopatológico. Na identificação de sorotipo viral, encontraram-se apenas os tipos DEN-1 e DEN-2 e um elevado número de casos ignorados ou em branco. Na Tabela 3 identifica-se o perfil clínico-epidemiológico de mulheres grávidas infectadas pelo vírus da dengue no Estado do Pará, no período de 2015 a 2019. 
Tabela 3. Perfil clínico-epidemiológico de mulheres grávidas infectadas pelo vírus da dengue no Estado do Pará, no período de 2015 a 2019.

\begin{tabular}{|c|c|c|}
\hline Variáveis & & SOS $=2.397$ \\
\hline Região de Saúde (CIR) de Residência & $\mathbf{N}^{\mathbf{0}}$ & $\%$ \\
\hline Araguaia & 56 & 2,3 \\
\hline Baixo Amazonas & 38 & 1,5 \\
\hline Carajás & 166 & 6,9 \\
\hline Lago de Tucuruí & 45 & 1,8 \\
\hline Metropolitana I & 110 & 4,5 \\
\hline Metropolitana II & 24 & 1 \\
\hline Metropolitana III & 47 & 1,9 \\
\hline Rio Caetés & 49 & 2 \\
\hline Tapajós & 34 & 1,4 \\
\hline Tocantins & 18 & 0,75 \\
\hline Xingu & 58 & 2,4 \\
\hline Marajó I & 4 & 0,16 \\
\hline Marajó II & 3 & 0,12 \\
\hline Classificação final & & \\
\hline Dengue clássico & 16 & 0,6 \\
\hline Febre hemorrágica do Dengue & - & - \\
\hline Dengue & 314 & 13 \\
\hline Dengue com sinais de alarme & 4 & 0,16 \\
\hline Dengue Grave & - & - \\
\hline Inconclusivo & 316 & 13,1 \\
\hline Ign/Branco & 228 & 9,5 \\
\hline Evolução do Paciente & & \\
\hline Cura & 308 & 12,8 \\
\hline Óbito & - & - \\
\hline Óbito pelo agravo & - & - \\
\hline Exame Sorológico (IgM) Dengue & & \\
\hline Positivo & 73 & 3,1 \\
\hline Negativo & 4 & 0,16 \\
\hline Inconclusivo & 8 & 0,3 \\
\hline Não realizado & 186 & 7,7 \\
\hline Exame sorológico ELISA & & \\
\hline Positivo & 10 & 0,41 \\
\hline Negativo & - & - \\
\hline Inconclusivo & - & - \\
\hline Não realizado & 272 & 11,3 \\
\hline
\end{tabular}




\begin{tabular}{lcc}
\hline Sorotipo de Dengue & 3 & 0,12 \\
DEN 1 & 3 & 0,12 \\
DEN 2 & - & - \\
DEN 3 & - & - \\
DEN 4 & - & \\
\hline
\end{tabular}

Fonte: Departamento de Informática do SUS/DATASUS.

\section{Discussão}

A dengue no Brasil se mostra como um dos principais agravos de saúde pública por se tratar de uma doença multifatorial e com rápida evolução. Nesse sentido, estudos que esclareçam os principais determinantes da dengue, principalmente os relacionados com a epidemiologia ecológica são extremamente úteis para uma melhor compreensão da doença e auxílio na criação de métodos de controle (Santana, 2018).

O presente estudo apresenta os casos notificados de dengue em gestantes no Estado do Pará, no período de 2015 a 2019. Nos últimos 5 anos, o Brasil registrou mais de 40 mil gestantes infectadas pelo vírus da dengue, fator que destaca a elevada carga da doença para grupos de risco que demandam atenção especial dos serviços de saúde. A incidência de dengue em gestantes acompanhou a ocorrência de casos na população geral em todas as regiões do país, e a letalidade por dengue nessa população foi superior à de mulheres em idade fértil não gestantes, com maior risco de óbito no terceiro trimestre de gestação (Nascimento, Siqueira, Coelho \& Júnior, 2017).

As maiores notificações de dengue em gestantes ocorreram nos meses de janeiro a maio, que são períodos que caracterizados por elevados índices pluviométricos na região estudada. Nesse contexto, em pesquisas realizadas em outras localidades como no Estado da Bahia, demonstrou que incidência da dengue e a ocorrência do vetor Aedes aegypti no período em estudo foram mais prevalentes em períodos chuvosos, uma vez esse fator, e outros como temperatura e umidade proporcionam condições ideais para a reprodução do vetor e a consequente proliferação dos casos de dengue (Costa \& Calado, 2016).

Destaca-se que houve redução significativa do número de casos de grávidas infectadas pelo vírus no Estado, que pode ser associado à implantação de medidas de controle pelo governo. Os programas governamentais deram início nos anos 90 com o Plano de Erradicação do Aedes aegypiti (PEAa), que preconiza ações multissetoriais para redução dos casos de dengue hemorrágica. No início da década de 2000, houve a implantação do Plano de Intensificação das Ações de Controle da Dengue (PIACD), e o Plano Nacional de Controle da Dengue (PNCD), pela probabilidade de aumento do risco de epidemias, a ocorrência de casos graves de dengue e a reintrodução e rápida disseminação do sorotipo 3 no Brasil (Zara et al., 2016).

Os casos de dengue apresentaram uma uniformidade quando se trata da idade gestacional, com discreto aumento no $2^{\circ}$ trimestre. Tais dados são semelhantes aos descritos anteriormente no estudo de Carles et al. na Guiana Francesa (onde também verificou-se uma maior ocorrência de infecção no $2^{\circ}$ trimestre, com valor de 40,9\%). O trimestre gestacional em que ocorre a infecção pelo vírus predispõe a ocorrência de desfechos adversos, uma vez que mães infectadas no primeiro trimestre da gestação têm um maior risco de aborto e quando a infecção acontece no último trimestre, há maiores riscos de baixo peso ao nascer, trabalho de parto prematuro e transmissão vertical (Alvarenga et al., 2009).

Quanto ao perfil clínico e epidemiológico, nota-se que as Regiões de Saúde do Carajás, Metropolitana I, Xingu, Araguaia e Rio Caetés obtiveram maior número de casos da doença. Esse achado corrobora com a pesquisa de incidência no Estado do Pará no período de 2003 a 2012, onde as Regiões do Carajás, Rio Caeté e Xingu foram as de maior incidência de dengue no Estado, demonstrando que a doença está associada principalmente aos fatores socioeconômicos das regiões (Chaves, Costa, Flores \& 
Bernardes, 2019).

Em relação à evolução do paciente, nota-se a cura da doença em parte das notificações e nenhum óbito foi registrado, esses achados se fazem pela crescente mobilização dos serviços de saúde ao longo dos anos principalmente em anos epidêmicos, fator que gera um aumento da demanda de atendimento precoce aos casos graves e, consequentemente, redução na letalidade pela doença (Oliveira, Araújo \& Cavalcanti, 2018).

Nos métodos de diagnóstico clínico-laboratorial, observa-se a utilização do exame sorológico (IgM) de dengue e ELISA, porém ressalta-se também o elevado número da não realização de exames laboratoriais. Os achados são semelhantes a outros estudos $23 \%$ foram confirmadas por laboratório e $77 \%$ pelo critério clínico/epidemiológico. O elevado número de notificações nos anos epidêmicos pode ter dificultado a realização de sorologias para o encerramento dos casos por laboratório (Feitoza, Koifman, Koifman \& Saraceni, 2017).

No que se refere às características virais, apesar dos determinantes para a ocorrência de formas graves da dengue não serem bem esclarecidos, todos os sorotipos são capazes de causar dengue grave. No entanto, o aumento de casos graves associados ao DENV2 sugere maior virulência desse sorotipo com eficiente replicação e alta carga viral, causando efeitos inflamatórios e tóxicos (Vicente et al., 2016).

\section{Considerações Finais}

Essa pesquisa demonstrou que a infecção por dengue em mulheres grávidas no Estado do Pará é uma realidade presente na região, e persiste como uma doença negligenciada. As complicações do estudo permearam um grande número de notificações ignoradas e em branco, que é um fator o qual revela a falta de completude dos dados e impacta diretamente na elaboração de possíveis intervenções de vigilância em saúde no Estado.

Observou-se que muitas grávidas não concluíram os exames de diagnóstico da doença, e também não tiveram o preenchimento completo da sua ficha notificação compulsória, sendo essas informações primordiais para as ações de prevenção e promoção da saúde direcionadas a esse público. Assim, enfatiza-se a necessidade de abranger a importância do conhecimento da doença pelos profissionais da saúde principalmente quando se trata de populações vulneráveis como as gestantes.

Ressalta-se a importância de mais estudos voltados à temática, para fornecer dados atualizados sobre as características sociodemográficas, clínicas e epidemiológicas do agravo, uma vez que a dengue pode trazer consequências que irão impactar diretamente na qualidade de vida da gestante. Assim, haverá a possibilidade de proporcionar estratégias que visem auxiliar na notificação e controle da doença na população em questão.

\section{Referências}

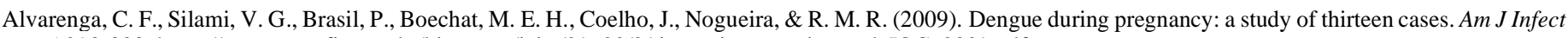
Dis; 5,298-303. https://www.arca.fiocruz.br/bitstream/icict/29620/2/ritamaria_nogueira_etal_IOC_2009.pdf

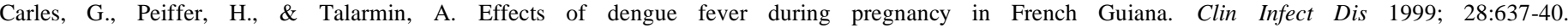
https://pubmed.ncbi.nlm.nih.gov/10194092/

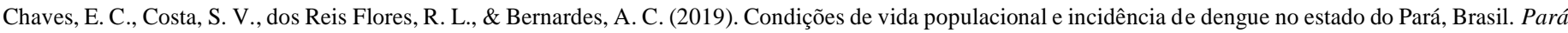
Research Medical Journal, 2(1-4). https://prmjournal.org/journal/prmjournal/article/doi/10.4322/prmj.2018.002

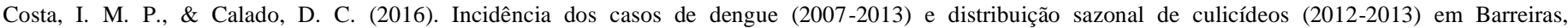
Bahia. Epidemiologia e Serviços de Saúde, 25, 735-744. https://www.scielosp.org/article/ress/2016.v25n4/735-744/.

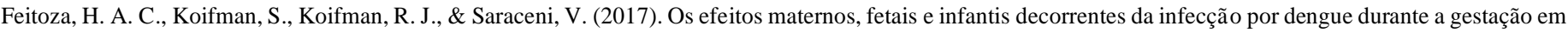
Rio Branco, Acre, Brasil, 2007-2012. Cadernos de Saúde Pública, 33, e00178915. https://www.scielosp.org/article/csp/2017.v33n5/e00178915/pt/.

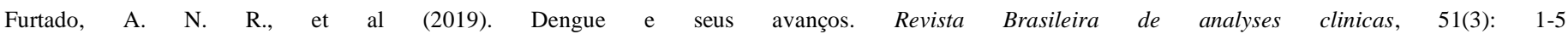
https://www.sumarios.org/artigo/dengue-e-seus-avan\%C3\%A7os 
Research, Society and Development, v. 10, n. 1, e20810111641, 2021 (CC BY 4.0) | ISSN 2525-3409 | DOI: http://dx.doi.org/10.33448/rsd-v10i1.11641

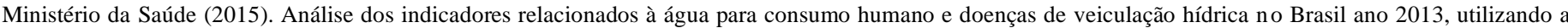

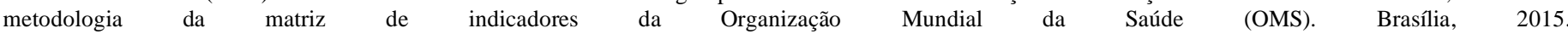
http://bvsms.saude.gov.br/bvs/publicacoes/analise_indicadores_agua_consumo_humano_doencas_hidrica_brasil_2013.pdf

Ministério da Saúde (2019). Secretaria de Vigilância em Saúde. Dengue no Brasil. http://saude.gov.br/vigilancia-em-saude


2007-2015. Epidemiologia e Serviços de Saúde, 26, 433-442. https://www.scielosp.org/article/ress/2017.v26n3/433-442/pt/.

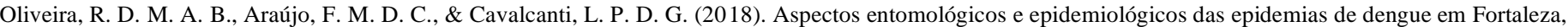
Ceará, 2001-2012. Epidemiologia e Serviços de Saúde, 27, e201704414. https://www.scielosp.org/article/ress/2018.v27n1/e201704414/pt/.

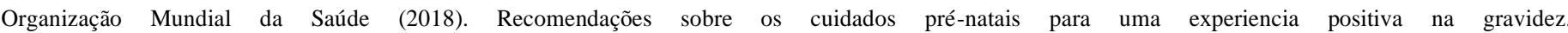
https://apps.who.int/iris/bitstream/handle/10665/2508 00/WHO-RHR-16.12-por.pdf?sequence=2\&isAllowed=y

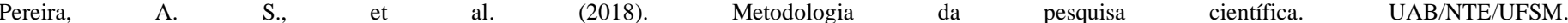
https://repositorio.ufsm.br/bitstream/handle/1/15824/Lic_Computacao_Metodologia-Pesquisa-Cientifica.pdf?sequence=1

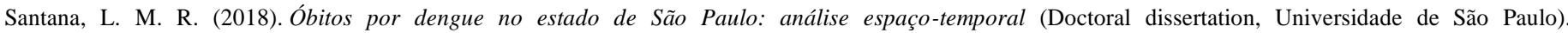
https://www.teses.usp.br/teses/disponiveis/6/6143/tde-06092018-121756/en.php

Sondo, K. A., et al. (2019). Infecção por dengue durante a gravidez em Burkina Faso: um estudo transversal. https://www.bvs.com.br.

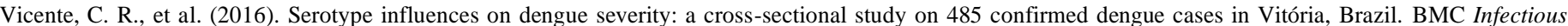
Diseases, 16(1), 320. https://pubmed.ncbi.nlm.nih.gov/27393011/.

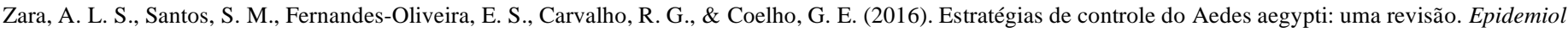
Serv Saude. 25(2), 391-404. https://www.scielo.br/scielo.php?pid=S2237-96222016000200391\&script=sci_abstract\&tlng=pt 